\title{
Consumo de Cigarrillo en Estudiantes de una Ciudad de Colombia: Factores Asociados por Género
}

\author{
Cigarette-smoking amongst students in a Colombian city: \\ gender-associated factors
}

\author{
Adalberto Campo-Arias ${ }^{1}$, Guillermo A. Ceballos ${ }^{2}$ y Edwin Herazo ${ }^{1}$
}

1Instituto Investigación del Comportamiento Humano, Bogotá.Colombia.

campoarias@comportamientohumano.org, eh@comportamientohumano.org

2 Facultad de Salud. Universidad del Magdalena. Colombia. gceballos@unimagdalena.edu.co.

Recibido 11 Noviembre 2008/Enviado para Modificación 6 Julio 2009/Aceptado 10 Julio 2009

\section{RESUMEN}

Objetivo Determinar los factores asociados por género al consumo de cigarrillo en estudiantes de secundaria en Santa Marta, Colombia.

Método Se diseñó un estudio transversal para conocer los factores asociados al consumo de cigarrillo durante el último mes (ocasional). Mediante regresión logística se ajustaron los factores relacionados con el consumo ocasional durante el último mes, para mujeres y para varones en forma independiente.

Resultados Un total de 2039 mujeres y 1536 varones participaron en el presente estudio. El $12 \%$ de los varones y el $8,1 \%$ de las mujeres informaron consumo ocasional de cigarrillo durante el último mes $(\mathrm{OR}=1,5)$. En mujeres, este hallazgo se relacionó con consumo de alcohol $(\mathrm{OR}=20,3)$, consumo de marihuana $(\mathrm{OR}=9,3)$, consumo de inhalante $(2,5)$, edad mayor de 15 años $(\mathrm{OR}=2,5)$, percepción de regular o pobre rendimiento académico $(\mathrm{OR}=1,9)$ y estudiar en colegio privado $(\mathrm{OR}=1,6)$. En varones, se relacionó con consumo de alcohol $(\mathrm{OR}=30,1)$, consumo de cannabis $(\mathrm{OR}=13,0)$, consumo de cocaína $(6,4)$, percepción de regular o pobre rendimiento académico $(\mathrm{OR}=2,7)$, orientación heterosexual $(\mathrm{OR}=2,4)$ y nivel socioeconómico alto $(\mathrm{OR}=2,0)$.

Conclusiones El consumo de cigarrillo se asocia diferencialmente en mujeres y varones. En mujeres, se relaciona especialmente con consumo de inhalantes, edad mayor de 15 años y estudiar en colegio privado; y en varones, con consumo de cocaína, orientación heterosexual y estrato socioeconómico alto.

Palabras Clave: Tabaquismo, estudiantes, adolescente, estudios transversales (fuente: DeCS, BIREME).

\section{ABSTRACT}

Objective Establishing gender-related factors associated with cigarette-smoking amongst adolescent students in Santa Marta, Colombia.

Method A cross-sectional study was designed to ascertain factors associated with lastmonth (occasional) cigarette-smoking. Logistic regression was used for independently 
adjusting factors associated with girls' and boys' last-month occasional cigarette-smoking. Results A total of 2,039 girls and 1,536 boys participated in the research. It was observed that $12.0 \%$ of boys and $8.3 \%$ of girls reported occasional cigarette-smoking during the last-month $(\mathrm{OR}=1.5)$. Cigarette-smoking in girls was strongly associated with drinking alcohol $(O R=20.4)$, cannabis use $(O R=9.3)$, inhalant use $(O R=2.5)$, being older than fifteen $(\mathrm{OR}=2.5)$, having poor or mediocre last month academic achievement $(\mathrm{OR}=1.9)$ and attending private schools $(\mathrm{OR}=1.6)$. Cigarette-smoking in boys was related to drinking alcohol $(O R=30.1)$, cannabis use $(O R=13.0)$, cocaine use $(O R=6.4)$, having poor or mediocre last month academic achievement $(\mathrm{OR}=2.7)$, having heterosexual orientation $(\mathrm{OR}=2.4)$ and having low socioeconomic status $(\mathrm{OR}=2.0)$.

Conclusions Cigarette-smoking is differentially associated in boys and girls. Cigarettesmoking in girls was related to inhalant consumption, being older than 15 and attending a private school; in boys, it was associated with cocaine use, having heterosexual orientation and low socioeconomic status.

Key Words: Smoking, student, adolescent, cross-sectional study (source: MeSH, NLM).

$\mathrm{E}$ 1 consumo de cigarrillo es la causa más importante de enfermedades que podrían prevenirse (1). Sin embargo, un número significativo de adolescentes inicia y se mantiene en el consumo de cigarrillo (2). Los adolescentes, por lo general, consumen cigarrillo de manera ocasional; sin embargo, un alto número evoluciona a un patrón de consumo diario (3). Algunos estudios sugieren que los fumadores ocasionales muestran menores riesgos psicosociales que los fumadores diarios y mayor facilidad de abandono del consumo (4).

En adolescentes, la prevalencia de consumo de cigarrillo varía según la forma como se defina el mismo. Los estudios informan que el consumo de cigarrillo por lo menos un día durante el último mes (consumo ocasional) se encuentra entre 5 y $40 \%$ (5-8). En tanto que la prevalencia de consumo de cigarrillo todos los días durante el último mes (consumo diario) es significativamente menor, aproximadamente entre 5 y $25 \%$, según la población estudiada (9-12).

Los datos disponibles muestran que el consumo de cigarrillo, por lo general, es más alto en varones que en mujeres adolescentes (11-16). Sin embargo, otras investigaciones presentan frecuencias similares en mujeres y en varones $(6,8,17)$ $\mathrm{y}$ algunas informan que el consumo es mayor en mujeres que en varones $(18,19)$.

Aunque existen pocos estudios que señalen los factores asociados a consumo de cigarrillo según el género, en forma independiente en mujeres y en varones. Los datos disponibles sugieren que algunos factores relacionados se comportan de manera distinta según el género. Por ejemplo, en mujeres se observa que el 
consumo de cigarrillo y otras sustancias puede ser una medida para controlar el peso (20); mientras que en varones el consumo de cigarrillo se observa con mayor frecuencia en los adolescentes que informan orientación sexual heterosexual (21).

Las campañas para la prevención del consumo de cigarrillo en adolescentes consideran estas diferencias entre mujeres y varones. Algunas investigaciones muestran diferencias por género en la preferencia por mensajes particulares utilizados en la prevención del consumo de cigarrillo (22). Es necesario promover el abandono del consumo de cigarrillo en adolescentes estudiantes considerando una perspectiva de género. En mujeres, el consumo de cigarrillotiene consecuencias negativas para patologías específicas como el cáncer de cuello uterino y repercusiones significativas durante el embarazo y para los hijos en el hogar (23).

El objetivo de este estudio fue investigar la prevalencia de consumo de cigarrillo durante el último mes y los factores relacionados según el género en estudiantes de secundaria de Santa Marta, Colombia.

\section{MÉTODO}

Para realizar este estudio analítico transversal se contó con permiso de las directivas de los colegios seleccionados y el consentimiento de los padres de familia o de los estudiantes participantes mayores de 18 años y el asentimiento de los participantes menores de edad. El comité de ética de la Facultad de Ciencias de la Salud de la Universidad del Magdalena aprobó este estudio. La investigación representaba un mínimo riesgo para los participantes (24). La participación fue enteramente voluntaria y se garantizó el completo anonimato.

Se realizó un muestreo probabilístico por conglomerados en el que se incluyeron todos los colegios públicos y privados de secundaria registrados en el Distrito Turístico, Cultural e Histórico de Santa Marta durante el año 2005. Se incluyeron instituciones localizadas en el área urbana y rural. Todos los colegios tenían la misma probabilidad de ser incluidos en la investigación.

Los participantes diligenciaron en el aula de clase un formulario autoadministrado que incluía incisos sobre los aspectos sociodemográficos, la percepción de rendimiento académico durante el último mes, la percepción de salud durante el último mes, el uso de alcohol y de alguna sustancia ilegal alguna vez en la vida y el consumo de cigarrillo (alguna vez en la vida, el consumo ocasional y el consumo diario durante el último mes). 
Se tomó para el análisis como variable dependiente haber fumado algún día durante el último mes (consumo ocasional) y como variables independientes se consideran la edad, orientación sexual, grado de escolaridad, tipo de colegio (público o privado), estrato socioeconómico, percepción de salud, percepción de rendimiento académico, consumo de sustancias legales (alcohol y pegantes) y consumo de sustancias ilegales (cannabis, cocaína e inyectables). Todas las variables cualitativas y cuantitativas se dicotomizaron cuando no era la condición natural.

Los datos se analizaron en STATA 9.0. Se hizo un análisis separado para mujeres y otro para varones. El análisis multivariado se realizó mediante regresión logística. Se determinaron razones de disparidad (OR) con intervalo de confianza del $95 \%$ (IC95\%). Se siguieron las recomendaciones de Greenland para el ajuste de las asociaciones. Al modelo final se le aplicó la prueba de HosmerLemeshow. Se aceptaron como significativos valores de $p<0,05$.

\section{RESULTADOS}

Participaron 2039 mujeres y 1536 varones. Las características de los participantes se muestran en la Tabla 1. La prevalencia global de consumo ocasional de cigarrillo durante el último mes fue $9,8 \%$ (IC95 \% 8,8-10,8) y la de consumo diario fue, 0,8 \% (IC95 \% 0,5-1,1). Se observó que 12,0 \% (IC95\% 10,4-13,6) de los varones y $8,1 \%$ (IC95 \% 6,9-9,3) de las mujeres informaron consumo ocasional de cigarrillo durante el último mes. La prevalencia fue significativamente mayor en varones que en mujeres (OR=1,5; IC95 \% 1,2-1,9; $\mathrm{p}<0,001)$.

En el análisis bivariado todas las variables estudiadas mostraron asociación significativa con consumo ocasional de cigarrillo durante el último mes, excepto la percepción de salud durante el último mes y el consumo alguna vez en la vida de sustancias ilegales inyectadas en mujeres, probablemente debido al bajo consumo de estas sustancias. Las OR de este análisis se presentan en las Tablas 2 y 3.

Para ajustar las asociaciones y controlar posibles variables confusoras se realizó un modelo multivariado mediante regresión logística. El mejor modelo final para mujeres y para varones se presenta en las Tablas 4 y 5 . 
Tabla 1. Características de estudiantes mujeres y varones de Santa Marta, Colombia

\begin{tabular}{|c|c|c|c|c|}
\hline \multirow{2}{*}{ Característica } & \multicolumn{2}{|c|}{ Mujeres } & \multicolumn{2}{|c|}{ Varones } \\
\hline & Frecuencia & Porcentaje & Frecuencia & Porcentaje \\
\hline Edad & & & & \\
\hline Menor de 15 años & 1672 & 82,0 & 1226 & 79,8 \\
\hline Mayor de 15 años & 367 & 18,0 & 310 & 20,2 \\
\hline Nivel académico & & & & \\
\hline Básica & 1494 & 73,3 & 1196 & 77,9 \\
\hline Media vocacional & 545 & 26,7 & 340 & 22,1 \\
\hline Estrato socioeconómico & & & & \\
\hline Bajo $(1,2$ y 3$)$ & 1652 & 81,0 & 1282 & 83,5 \\
\hline Alto $(4,5$ y 6$)$ & 387 & 19,0 & 254 & 16,5 \\
\hline Colegio & & & & \\
\hline Privado & 716 & 35,1 & 650 & 42,3 \\
\hline Público & 1323 & 64,9 & 886 & 57,7 \\
\hline Orientación sexual & & & & \\
\hline Heterosexual & 1676 & 82,2 & 1332 & 86,7 \\
\hline No heterosexual & 383 & 17,8 & 204 & 13,3 \\
\hline Rendimiento académico & & & & \\
\hline Excelente-bueno & 1685 & 82,6 & 1216 & 79,2 \\
\hline Regular-malo & 354 & 17,4 & 320 & 20,8 \\
\hline Percepción de salud & & & & \\
\hline Excelente-bueno & 1652 & 81,0 & 1297 & 84,4 \\
\hline Regular-malo & 387 & 19,0 & 239 & 15,6 \\
\hline Alcohol & & & & \\
\hline Sí & 1152 & 56,5 & 1129 & 73,5 \\
\hline No & 887 & 43,5 & 407 & 26,5 \\
\hline Marihuana & & & & \\
\hline Sí & 24 & 1,2 & 46 & 3,0 \\
\hline No & 2015 & 98,8 & 1490 & 97,0 \\
\hline Cocaína & & & & \\
\hline Sí & 5 & 0,2 & 27 & 1,8 \\
\hline No & 2034 & 99,8 & 1509 & 98,2 \\
\hline Pegantes & & & & \\
\hline Sí & 81 & 96,0 & & 6,3 \\
\hline No & 1958 & 4,0 & 1439 & 93,7 \\
\hline Inyectables & & & & \\
\hline Sí & 2038 & 100,0 & 16 & 1,0 \\
\hline No & 1 & 0 & 1520 & 99,0 \\
\hline
\end{tabular}

En el presente estudio se observa que el consumo ocasional de cigarrillo durante el último mes es mayor en varones que en mujeres. Tanto en mujeres como en varones se presenta asociación estadísticamente significativa con consumo de alcohol y marihuana y pobre o regular rendimiento académico. Adicionalmente, en mujeres, las variables relacionadas son consumo de inhalantes (pegantes), edad mayor de 15 años y estudiar en colegio privado; $y$ en varones, las variables asociadas son consumo de cocaína, orientación sexual heterosexual y estrato socioeconómico alto. 
Tabla 2. Análisis bivariado para consumo ocasional de cigarrillo durante el último mes en 2039 mujeres estudiantes de Santa Marta, Colombia

\begin{tabular}{lcc}
\hline \multicolumn{1}{c}{ Variable } & OR & IC95 \% \\
\hline Edad mayor de 15 años & 4,3 & $3,1-5,9$ \\
Media vocacional & 3,1 & $2,2-4,3$ \\
Estrato socioeconómico alto & 1,4 & $1,4-2,9$ \\
Colegio privado & 3,1 & $2,3-4,3$ \\
Orientación sexual heterosexual & 1,8 & $1,1-3,1$ \\
Regular o malo rendimiento académico & 2,7 & $1,9-3,8$ \\
Regular o malo percepción de salud & 1,4 & $0,93-2,0$ \\
Experiencia con alcohol & 28,5 & $11,6-69,6$ \\
Experiencia con cannabis & 30,6 & $12,5-75,1$ \\
Experiencia con cocaína & 46,5 & $5,2-418,8$ \\
Experiencia con inhalantes & 3,5 & $2,0-6,1$ \\
\hline
\end{tabular}

Tabla 3. Análisis bivariado para consumo ocasional de cigarrillo durante el último mes en 1536 varones estudiantes de Santa Marta, Colombia

\begin{tabular}{lcc}
\hline \multicolumn{1}{c}{ Variable } & OR & IC95 \% \\
\hline Edad mayor de 15 años & 2,8 & $2,1-4,0$ \\
Media vocacional & 2,7 & $1,9-3,7$ \\
Estrato socioeconómico alto & 2,3 & $1,6-3,3$ \\
Colegio privado & 2,6 & $1,9-3,6$ \\
Orientación sexual heterosexual & 1,8 & $1,1-3,6$ \\
Regular o malo rendimiento académico & 3,2 & $2,3-4,4$ \\
Regular o malo percepción de salud & 1,9 & $1,3-2,7$ \\
Experiencia con alcohol & 38,9 & $9,6-157,6$ \\
Experiencia con cannabis & 25,3 & $12,8-49,9$ \\
Experiencia con cocaína & 23,4 & $9,8-56,3$ \\
Experiencia con inhalantes (pegantes) & 4,2 & $2,6-6,6$ \\
Experiencia con sustancias inyectadas & 34,2 & $9,6-121,2$ \\
\hline
\end{tabular}

Tabla 4. Modelo multivariado para consumo ocasional de cigarrillo durante el último mes en 2039 mujeres estudiantes de Santa Marta, Colombia

\begin{tabular}{lcc}
\hline \multicolumn{1}{c}{ Variable } & OR & IC95 \% \\
\hline Experiencia con alcohol & 30,1 & $7,0-129,0$ \\
Experiencia con cannabis & 13,0 & $6,0-28,4$ \\
Experiencia con cocaína & 6,4 & $2,1-19,6$ \\
Rendimiento académico pobre o regular & 2,7 & $1,9-3,8$ \\
Orientación heterosexual & 2,4 & $1,2-4,8$ \\
Estrato socioeconómico alto & 2,0 & $1,4-3,0$ \\
\hline Bondad del ajuste, prueba de Hosmer-Lemeshow $\mathrm{X}^{2}=4,3 ; \mathrm{gl}=4 ; \mathrm{p}=0,595$ &
\end{tabular}


Tabla 5. Modelo multivariado para consumo ocasional de cigarrillo durante el último mes en 1536 varones estudiantes de Santa Marta. Colombia

\begin{tabular}{lcc}
\multicolumn{2}{c}{ último mes en 1536 varones estudiantes de Santa Marta. Colombia } \\
\hline \multicolumn{1}{c}{ Variable } & OR & IC95 \% \\
\hline Experiencia con alcohol & 30,1 & $7,0-129,0$ \\
Experiencia con cannabis & 13,0 & $6,0-28,4$ \\
Experiencia con cocaína & 6,4 & $2,1-19,6$ \\
Rendimiento académico pobre o regular & 2,7 & $1,9-3,8$ \\
Orientación heterosexual & 2,4 & $1,2-4,8$ \\
Estrato socioeconómico alto & 2,0 & $1,4-3,0$ \\
\hline
\end{tabular}

Bondad del ajuste, prueba de Hosmer-Lemeshow $X^{2}=4,9 ; g l=4 ; p=0,558$

En el presente estudio se observa que el 8,1\% de las mujeres presentaron consumo ocasional de cigarrillo durante el último mes. Este dato es similar a lo documentado en otras investigaciones $(14,15)$. Sin embargo, la cifra es significativamente superior a la hallada por otros investigadores (11, 15, 27); pero menor a la encontrada en otros estudios $(6-8,9,13,17)$. Por su parte, esta investigación muestra que el $12,0 \%$ de los varones son fumadores ocasionales. El porcentaje es comparable a lo informado en otras investigaciones $(9,11,13)$. No obstante, el dato supera lo observado en otros estudios $(7,8,15-17)$ e inferior a lo documentado en otros contextos $(6,14)$. Asimismo, en el presente estudio se corrobora la mayor prevalencia de consumo ocasional de cigarrillo en el grupo masculino. No obstante, la magnitud de la asociación es débil. Un gran número de estudios muestra que los varones fuman con mayor frecuencia que las mujeres (7,11-16). Otras investigaciones presentan similares prevalencias en mujeres y en varones $(6,8,9,17)$ y en algunos países o sociedades la prevalencia en mujeres supera la prevalencia hallada en varones $(18,19)$.

En el presente estudio se encontró que la edad se relacionaba significativamente con el consumo de cigarrillo durante el último mes sólo en mujeres. Por lo general, la mayoría de los estudios en población adolescente informan, tanto en varones como en mujeres, que la prevalencia de consumo se incrementa con edad $(9,11,13)$.

Los datos presentados indican que en mujeres y varones el consumo de cigarrillo es independiente del nivel de escolaridad. La información es concordante con los hallazgos de Horta et al., quienes encontraron que el consumo de cigarrillo en adolescentes brasileños era independiente del grado de escolaridad (13). Contrario a este dato, Malcon et al., observaron que el consumo de cigarrillo era mayor en adolescentes con menor escolaridad (9). Arillo-Santillan et al., informaron que la prevalencia de consumo era superior en estudiantes de media vocacional (high school) que en estudiantes de básica secundaria (middle-school) (11) y Wallace et al., y Lewis et al., hallaron que el consumo era más frecuente en los grados superiores $(6,17)$. 
En la investigación que se presenta se observó que el mayor nivel socioeconómico se relacionaba con el consumo de cigarrillo sólo en el grupo masculino. Divergente con esta observación, Arillo-Santillan et al., mostraron que el mayor número de fumadores se encontraban en los estratos más altos tanto en varones como en mujeres (11) y Lewis et al., y Xue et al., notaron que el consumo era más frecuente en los estratos menos favorecidos $(17,25)$.

Con relación al tipo de colegio, en el presente informe se encontró que estudiar en colegio privado se asociaba de manera significativa a consumo de cigarrillo únicamente en mujeres. Similar a este hallazgo, en mujeres y en varones, Gong et al., y Valois et al., informaron que los estudiantes de colegios privados tenían más probabilidades que los estudiantes de colegios públicos de ser fumadores $(14,26)$. No obstante, Glanz et al., mostraron que el consumo de cigarrillo era mayor en estudiantes que asistían a colegios públicos (27) y Fraga et al., observaron que la frecuencia de consumo de cigarrillo era similar en colegios públicos y privados, tanto en mujeres y como en varones (15).

En lo que respecta a la orientación sexual, en la presente investigación se observó que la orientación heterosexual se relacionó con el consumo de cigarrillo únicamente en el grupo masculino. Este dato es similar al observado por Griensven et al., en adolescentes tailandeses (21). Asimismo, Austin et al., documentaron que el riesgo de consumo de cigarrillo era mayor en varones heterosexuales; sin embargo, observaron que las mujeres no heterosexuales mostraban un riesgo incrementado comparada con las heterosexuales (28). Russell et al., informaron que los adolescentes homosexuales fumaban un menor número de cigarrillo por día que los bisexuales y heterosexuales (29).

En la relación con la percepción de salud, en el análisis que se presenta no se observó relación entre percepción de salud durante el último mes y consumo de cigarrillo. En forma similar, Campo-Arias et al., observaron que el consumo de cigarrillo era independiente de la percepción de salud en estudiantes de media vocacional (30).

En el presente estudio se encontró que la percepción de rendimiento académico regular o malo se relacionó con consumo de cigarrillo sólo en el grupo femenino. Esta información es consistente con lo observado en estudios precedentes con estudiantes de ambos sexos. Arillo-Santillan et al., encontraron en estudiantes de colegios públicos que el consumo de cigarrillo guardaba asociación significativa con menores logros académicos (10). De la misma forma, Aloise-Young et al., observaron que los adolescentes fumadores mostraban menor desempeño 
académico que los no fumadores (31), y Campo-Arias et al., hallaron que estudiantes de media vocacional que fumaban informaron menor percepción de rendimiento académico (32).

En relación con el consumo de sustancias legales, en este estudio se observa una fuerte asociación entre el consumo de alcohol y el consumo de cigarrillo, tanto en mujeres como en varones; mientras que el consumo de inhalantes se relacionó con el consumo de cigarrillo únicamente en mujeres. La asociación entre consumo de alcohol y consumo de cigarrillo es consistente en la mayoría de los estudios en adolescentes $(11,33)$. En mujeres y varones, Muilenburg y Johnson observaron que el consumo de inhalantes se relacionaba significativamente con el consumo de cigarrillo (34).

En relación con el consumo de sustancias ilegales se observó que el consumo de marihuana se asoció a consumo de cigarrillo en varones y en mujeres; sin embargo, el consumo de cocaína sólo fue significativo en varones. Por lo general, el consumo de sustancias ilegales, en ambos sexos, guarda una significativa asociación con el consumo de cigarrillo en la mayoría de las poblaciones de adolescentes (11).

Los hallazgos del presente estudio sugieren que efectivamente la brecha actual en la diferencia de frecuencia de consumo de cigarrillo es estrecha en mujeres y varones y que las variables relacionadas con el consumo de cigarrillo presentan un patrón característico que pueden ser mejor comprendidas desde una perspectiva de género. Dado los cambios sociales y culturales de las últimas décadas se observa un incremento del consumo de cigarrillo en mujeres (22). Este hecho ha creado un nuevo sector de mercado en el que se promocionan productos específicos para el público femenino (23). De igual manera, es posible que estas diferencias no se hayan observado en algunos estudios porque no se había realizado un análisis por separado para mujeres y varones. El consumo de cigarrillo es un problema de salud pública en el que interactúan de manera compleja variables constitucionales y medioambientales (35). Por ejemplo, en adolescentes, la exposición a comerciales que promueven el consumo de cigarrillo varía según la legislación de cada país (36). Esta multiplicidad de factores puede explicar las variaciones en la prevalencia y las asociaciones por género en los diferentes países, culturas o contextos (17).

Las intervenciones dirigidas a prevenir el consumo de cigarrillo y aquellas orientadas a promover su abandono deben considerar siempre los aspectos relacionados con el género. En mujeres en edad fértil, es necesario considerar 
que el consumo de cigarrillo se asocia a consecuencias negativas para la madre y para el fruto del embarazo (mayor riesgo de parto pretérmino y neonatos de bajo peso) (23). Además, algunos estudios sugieren que las mujeres mienten con más frecuencia acerca del consumo de cigarrillo que los varones (37) y que las mujeres presentan más dificultad para abandonar el consumo de cigarrillo que los varones (22).

Es muy importante el papel activo que deben jugar todos los profesionales de la salud, médicos generales, médicos familiares, pediatras, enfermeras, entre otros, en la promoción de un estilo de vida saludable desde las primeras consultas en niños, niñas y adolescentes, esto debe incluir la prevención del inicio del consumo de cigarrillo (38). De la misma forma, es necesaria la evaluación clínica rutinaria del consumo de cigarrillo con el propósito de promover decididamente el abandono del consumo antes de que se establezca un patrón de consumo diario de cigarrillo. El consumo diario de cigarrillo incrementa la posibilidad de presentar dependencia a la nicotina y mayor dificultad para el abandono sostenido y prolongado del consumo de cigarrillo (39).

El presente estudio presenta la fortaleza de analizar el consumo de cigarrillo y los factores asociados al mismo en una muestra grande y representativa de estudiantes de una ciudad del Caribe colombiano y realizar un análisis multivariado por género. Sin embargo, presenta la limitación propia de un estudio transversal que no permite evaluar la dirección de causalidad de la asociación. De la misma forma, no se investigaron otras variables que en estudios precedentes se han relacionado con el consumo de cigarrillo. Asimismo, podría considerarse que el autoinforme de consumo de cigarrillo es poco confiable. Sin embargo, diferentes estudios con adolescentes muestran que es alta la confiabilidad del informe de consumo de cigarrillo mediante encuestas autodiligenciadas (40).

Se concluye que el consumo de cigarrillo presenta un patrón diferencial para mujeres y varones. El consumo de cigarrillo es un poco mayor en varones que en mujeres. En mujeres, se relaciona en forma importante con el consumo de inhalantes, edad mayor de 15 años y asistir a colegio privado; y en varones, con el consumo de cocaína, orientación sexual heterosexual y estrato socioeconómico alto. Se requiere de más investigación en esta área

Agradecimientos. Este trabajo lo financió Fonciencias de la Universidad del Magdalena y la Universidad Autónoma de Bucaramanga mediante el Convenio 030 de 2006. 


\section{REFERENCIAS}

1. Mokdad AH, Marks JS, Stroup DF, Gerberding JL. Actual causes of death in the United States, 2000. JAMA 2004; 291: 1238-1245.

2. Jackson C, Dickinson D. Cigarette consumption during childhood and persistence of smoking through adolescence. Arch Pediatr Adolesc Med 2004; 158: 1050-1056.

3. Lindström M, Isacsson SO. Malmö Shoulder-Neck Study Group. Long term and transitional intermittent smokers: a longitudinal study. Tob Control 2002; 11: 61-67.

4. Duhig AM, Caballo DA, McKee SA, George TP, Krishnan-Sarin S. Daily patterns of alcohol, cigarette, and marijuana use in adolescent smokers and nonsmokers. Addictive Behav 2005; 30: 271-283.

5. Encuesta mundial sobre tabaquismo en la juventud: resultados en las Américas. Bol Epidemiol OPS 2002; 23: 6-14.

6. Wallace JM, Bachman JG, O'Malley PM, Schulenberg JE, Cooper SM, Johnston LD. Gender and ethnic differences in smoking, drinking and illicit drug use among American 8th, 10th and 12th grade students, 1976-2000. Addiction 2003; 98: 225-234.

7. Eaton DK, Kann L, Kinchen S, Ross J, Hawkins J, Harris WA, et al. Youth risk behavior surveillance-United States, 2005. MMWR Surveill Summ. 2006; 55: 1-108.

8. Weisner C, Peñaranda D. Encuesta mundial de tabaquismo en jóvenes. Reporte de Bogotá, Colombia. Rev Colomb Cancerol 2002; 6: 5-14.

9. Malcon MC, Menezes AMB, Chatkin M. Prevalência e fatores de risco para tabagismo em adolescentes. Rev Saude Publica 2003; 37: 1-7.

10. Martínez JA, Amaya W, Campillo HA, Campo A, Díaz LA. Factores asociados con el consumo diario de cigarrillo en adolescentes estudiantes de básica secundaria de Bucaramanga, Colombia. Biomédica 2005; 25: 518-528.

11. Arillo-Santillan E, Lazcano-Ponce E, Hernandez-Avila M, Fernández E, Allen B, et al. Association between individual and contextual factors and smoking in 13,293 Mexican students. Am J Prev Med 2005; 28: 41-51.

12. Martínez-Mantilla JA, Amaya-Naranjo W, Campillo HA, Díaz-Martínez LA, Campo-Arias A. Daily cigarette smoking among Colombian high school students: gender related psychosocial factors. Rev Latinoamer Enfermagem 2008; 16: 903-907.

13. Horta B, Calheiros P, Pinheiro R, Tomasi E, Costa K. Tabagismo em adolescentes de área urbana na região Sul do Brasil. Rev Saude Publica 2001; 35: 159-164.

14. Gong J, Chen X, Guo Q, Zhou D, Palmer PH, Zhang D, et al. Are private school students more likely to smoke than public school students in China? Prev Med 2006; 43: 117-121.

15. Fraga $\mathrm{S}$, Ramos $\mathrm{E}$, Barros $\mathrm{H}$. Uso de tabaco por estudantes adolescentes portugueses e fatores associados. Rev Saude Publica 2006; 40: 620-626.

16. Granero R, Sánchez M. Cambios en el uso de tabaco y factores relacionados en estudiantes del sexto al noveno grado, Estado Lara, Venezuela, años 2002 y 2003. Cad Saude Publica 2006; 22: 1893-1899.

17. Lewis PC, Harrell JS, Bradley C, Deng S. Cigarette use in adolescents: The cardiovascular health in children and youth study. Res Nurs Health 2001; 24: 27-37.

18. Eptein JA, Botvin GJ, Diaz T. Ethnic and gender differences in smoking prevalence among a longitudinal sample of inner-city adolescents. J Adolesc Health 1998; 23: 160-166.

19. Pinilla J, González B, Barber P, Santana Y. Smoking in young adolescents: an approach multilevel discrete choice models. J Epidemiol Community Health 2002; 56: 227-232.

20. Baus J, Kupek E, Pires M. Prevalência e fatores de risco relacionados ao uso de drogas entre escolares. Rev Saude Publica 2006; 36: 40-46.

21. Griensven F, Kilmarx PH, Jeeyapantt S, Manopaiboon C, Korattana S, Jenkins RA, et al. The prevalence of bisexual and homosexual and related health risks among adolescents in Northern Thailand. Arch Sex Behav 2004; 33: 137-147. 
22. Flynn BS, Worden JK, Bunn JY, Dorwaldt AL, Connolly SW, Ashikaga T. Youth audience segmentation strategies for smoking-prevention mass media campaigns based on message appeal. Health Educ Behav 2007; 34: 578-593.

23. Mackay J, Amos A. Women and tobacco. Respirology 2003; 8: 123-130.

24. Resolución 008430, por la cual se establecen las normas científicas, técnicas y administrativas para la investigación en salud. Santafé de Bogotá: Ministerio de Salud; 1993.

25. Xue Y, Zimmerman MA, Cadwell $\mathrm{CH}$. Neighborhood residence and cigarette smoking among urban youth: the protective role of prosocial activities. Am J Public Health 2007; 97: 1865-1872.

26. Valois RF, Thatcher WG, Drane JW, Reinenger BM. Comparison of selected health risk behaviors between adolescents in public and private high schools in South Carolina. J Sch Health 1997; 67: 434-440.

27. Glanz K, Mau M, Steffen A, Maskarinec G, Arriola KJ. Tobacco use among Native Hawaiian middle school students: its prevalence, correlates and implications. Ethn Health 2007; 12: 227-244.

28. Austin SB, Ziyadeh N, Fisher LB, Kanh JA, olditz GA, Frazier AL. Sexual orientation and tobacco use in a cohort study of US adolescent girls and boys. Arch Pediatr Adolesc Med 2004; 158: 317-322.

29. Russell ST, Driscoll AK, Troung N. Adolescent same-sex romantic attractions and relationships: implications for substance use and abuse. Am J Public Health 2002; 92: 198-202.

30. Campo-Arias A, Dallos CM, González SJ, Rodríguez DC, Sánchez ZM, Díaz LA. Consumo de cigarrillo y síntomas depresivos en estudiantes de Bucaramanga, Colombia. Arch Argent Pediatr 2007; 105: 12-16.

31. Aloise-Young PA, Cruickshank C, Chavez EC. Cigarette smoking and perceived health in school dropouts: A comparison of Mexican American and Non-Hispanic white adolescents. J Pediatr Psychol 2002; 27: 497-507.

32. Campo-Arias A, González SJ, Sánchez ZM, Rodríguez DC, Dallos CM, Díaz-Martínez LA. Percepción de rendimiento académico y síntomas depresivos en estudiantes de media vocacional de Bucaramanga, Colombia. Arch Pediatr Uruguay 2005; 76: 44-49.

33. Rodríguez DC, Dallos CM, González SJ, Sánchez ZM, Díaz-Martínez LA, Rueda-Jaimes GE, et al. Asociación entre síntomas depresivos y consumo abusivo de alcohol en estudiantes de Bucaramanga, Colombia. Cad Saude Publica 2005; 21: 1402-1407.

34. Muilenburg JL, Johnson WD. Inhalant use and risky behavior correlates in a sample of rural middle school students. Subst Abus 2006; 27: 21-25.

35. Hettema JM, Corey LA, Kendler KS. A multivariate genetic analysis of the use of tobacco, alcohol, and caffeine in a population based sample of male and female twins. Drug Alcohol Depend 1999; 57: 69-78.

36. Forster JL, Widome R, Bernat DH. Policy interventions and surveillance as strategies to prevent tobacco use in adolescents and young adults. Am J Prev Med 2007; 33 (Suppl. 6): S335-339.

37. Sarraf-Zadegan N, Boshtam M, Shahrokhi S, Naderi GA, Asgary S, Shahparian M, et al. Tobacco use among Iranian men, women and adolescents. Eur J Public Health 2004; 14: 76-78.

38. Gregorio DI. Counseling adolescent for smoking prevention: A survey of primary care physicians and dentists. Am J Public Health 1994; 84: 1151-1153.

39. Gervais A, O'Loughlin J, Meshefedjian G, Bancej C, Tremblay M. Milestone in the natural course of onset of cigarette use among adolescents. CMAJ 2006; 175: 255-261.

40. Post A, Gilljam H, Rosedahl I, Meurling L, Bremberg S, Galanti MR. Validity of self reports in a cohort of Swedish adolescent smokers and smokeless tobacco (snus) users. Tob Control 2005; 14: 114-117. 